\title{
Welche Fortbildungsmedien steigern die Qualität der ärztlichen Leistungserbringung?*
}

\section{Max Giger}

Präsident der Kommission für Weiter- und Fortbildung (KWFB) der FMH
* Der vorliegende Text basiert auf einem Vortrag, den der Autor im Rahmen einer Einladungsveranstaltung des Schweizerischen Ärzteverlags EMH am 5. Juni 2008 in Zürich hielt.

Korrespondenz:

Dr. med. Max Giger

Rosenrain 9

$\mathrm{CH}-8400$ Winterthur

max.giger@hin.ch
Die Fortbildung gehört zu den Berufspflichten aller Ärztinnen und Ärzten. Diese Ausführungen sollen in knapper Form einen Überblick vermitteln über folgende Aspekte dieser Thematik:

- Fortbildung im Allgemeinen:

- gesetzliche Grundlagen,

- Inhalte,

- Formen,

- Anerkennung;

- Welche Formen beeinflussen Ärztinnen und Ärzte?

- Welche Medien können eingesetzt werden?

\section{Gesetzliche Grundlagen}

Das Medizinalberufegesetz hält die Fortbildungspflicht im Art. 40 Abs. b wie folgt fest:

Die Ärzte vertiefen, erweitern und verbessern ihre beruflichen Kenntnisse, Fähigkeiten und Fertigkeiten durch lebenslange Fortbildung.

Bei Nichterfüllen der Fortbildungspflicht sieht das Medizinalberufegesetz im Artikel 43 Disziplinarmassnahmen - von der Verwarnung bis zu einer Busse von 20000 Franken - vor. Die Kontrolle obliegt seit dem 1. September 2007 den Kantonen. Deshalb hat die Ärztekammer im Dezember 2007 eine entsprechende Änderung der Fortbildungsordnung verabschiedet. Es besteht kein Kontrollzwang mehr. Die FMH bzw. die Fachgesellschaften händigen auf Verlangen bei Erfüllen der selbstverantworteten Fortbildung von 50 Credits pro Jahr ein Fortbildungsdiplom aus. Nur ein verschwindend kleiner Anteil - zwei bis drei Prozent - der Ärztinnen und Ärzte genügen diesen Vorschriften nicht.

\section{Inhalt der Fortbildung}

Der Inhalt der Fortbildung wird einerseits durch die Ziele der Aus- und Weiterbildung gemäss Artikel 6, 7 und 17 des Medizinalberufegesetzes und andererseits durch die aktuelle Art der Berufsausübung gegeben. Die folgenden Punkte seien hier besonders erwähnt:

- Stellen von sicheren Diagnosen und Verordnen bzw. Durchführen der geeigneten Therapien;

- Respektieren der Menschenwürde;

- Begleiten der Patientinnen und Patienten bis zum Lebensende;
- selbständiges Handeln in Notfallsituationen;

- wirtschaftlicher Einsatz der zur Verfügung stehenden Mittel;

- Grenzen der medizinischen Tätigkeit erkennen und respektieren;

- Verstehen der ethischen Dimensionen des beruflichen Handelns und Wahrnehmen von Verantwortung gegenüber Individuum und Gesellschaft.

Es werden Sozialkompetenz und Verantwortung gefordert. Diese gilt es besonders zu entwickeln. Das bedeutet, dass der Kommunikation mehr Gewicht beigemessen werden sollte.

Die Fortbildungsordnung der FMH regelt den Aufbau der Fortbildungsprogramme der Fachgesellschaften. Diese schreiben in den Fortbildungsprogrammen die Kernfortbildung vor; diese umfasst 25 Credits. Die restlichen Credits sind von den Fortbildungspflichtigen entsprechend ihren individuellen fachlichen Interessen und der aktuellen Berufstätigkeit zusammenzustellen. Im Allgemeinen empfiehlt sich ein systematischer Aufbau der Fortbildung anhand der folgenden Schritte:

- Erkennen eines Defizits an Wissen und Können;

- Festsetzung eines Zielpunktes für die Verbesserung;

- Auswahl der Lernmethode;

- Sichtung des Fortbildungsangebotes;

- Erarbeitung des Lernziels;

- Selbstkontrolle des Lernerfolgs;

- Nutzung des Zuwachses von Wissen und Können in der praktischen Arbeit;

- kontinuierliche Überprüfung des Wissens und Könnens;

- besonderes Gewicht ist der Entwicklung der ärztlichen Haltung als kontinuierlichem Prozess beizumessen.

Der Umfang der Fortbildung richtet sich nach dem Fortbildungsbedürfnis jeden Arztes. Im Allgemeinen bilden sich unsere Kolleginnen und Kollegen während zweier Wochen pro Jahr oder einer Stunde pro Woche fort.

Als Mittel für die Fortbildung stehen Präsenzveranstaltungen, Printmedien und E-LearningProgramme zur Verfügung. 


\section{Anerkennung von Fortbildungs- veranstaltungen}

Die Anerkennung der Fortbildungsveranstaltungen erfolgt nach den Richtlinien der Schweizerischen Akademie der Medizinischen Wissenschaften. Die Mitglieder der FMH sind aufgrund der Standesordnung verpflichtet, diese Richtlinien zu befolgen. Dadurch werden eine hohe Qualität der Fortbildungen und vor allem Unabhängigkeit der Fortzubildenden angestrebt. Auch sollte das Ziel der finanziellen Unabhängigkeit verfolgt werden. Mit anderen Worten: Die Ärztinnen und Ärzte sollten ihre Fortbildung möglichst selbst finanzieren. Dies wird über kurz oder lang zu einer Flurbereinigung im Fortbildungssektor führen: Die Zahl der Veranstaltungen wird zurückgehen, deren Qualität wird zunehmen.

Die Anerkennung von Fortbildungsinhalten wird von den einzelnen Fachgesellschaften sehr unterschiedlich gehandhabt. Die Spanne reicht von der Anerkennung praktisch jeder für die ärztliche Tätigkeit nützlichen - gemäss den SAMWRichtlinien unabhängigen - Veranstaltung bis zur expliziten Nennung von praktisch-wissenschaftlich hochkarätigen Fortbildungskursen. Bezüglich der Effizienz der Veranstaltungen schweigen die Fortbildungsprogramme.

\section{Verhaltensänderung durch Fortbildung?}

Fortbildung soll das Verhalten ändern, sie soll zu einer Optimierung der Betreuung und Behandlung der Patienten führen. Die Frage darf gestellt werden: Was bringen 80 bis 100 Stunden jährlicher Fortbildung? Die Literatur darüber ist spärlich.

Die Cochrane Collaboration Review 2007 kann wie folgt zusammengefasst werden:

- interaktive Workshops können zu mässigen Änderungen in der Leistungserbringung führen. Lehrveranstaltungen alleine führen praktisch nicht zu Verhaltensänderungen;

- interaktive Erziehungsworkshops können zu mässigen Verhaltensänderungen führen;

- Vorlesungen und Präsentationen alleine führen kaum zu Praxisänderungen.

So viel über die Form der Fortbildungen. Über deren Auswirkung auf die Patientenbetreuung liegen keine Cochrane Reviews vor. Hier sei auf unsere eigenen Erfahrungen hingewiesen:

- In der Behandlung der Hypertonie gilt immer noch die Dreierregel: $1 / 3$ korrekt behandelt, $1 / 3$ nicht behandelt, $1 / 3$ falsch behandelt;

- seit den Siebziger Jahren weiss man, dass nur selten eine Indikation zur Differenzierung der Leukozyten notwendig ist. Man vergegenwär- tige sich, wie oft diese Leistung noch erbracht wird - mit welchem Ziel?

- in den Achtzigerjahren initiierte die FMH ein Projekt «Back in Time». Rückenbeschwerden bedürfen primär nach einer klinischen Untersuchung keiner Spezialuntersuchung. Man halte sich vor Augen, wie oft schon nach drei Tagen ein CT oder MRI, selbst bei gut Zwanzigjährigen, veranlasst wird - mit welchem Ziel?

Ich schliesse daraus, dass es mindestens eine Generation braucht, bis Verhaltensänderungen in den ärztlichen Alltag Eingang finden.

Die individuellen Fortbildungsbedürfnisse zu bestimmen ist schwierig. Es stehen heute folgende Methoden zur Verfügung:

- Selfassessment (z.B. Multiple Choice, Kurzantwortfragen, OSCE);

- Peerassessment;

- Fortbildungsportfolio.

Diese Methoden sollen im Laufe der nächsten zwei Jahre zur breiteren Anwendung gebracht werden, dies mit Unterstützung des Instituts für Medizinische Lehre (IML) der Universität Bern.

\section{Medien in der Fortbildung}

Die folgenden Medien gelangen in der Fortbildung zum Einsatz:

- Printmedien:

- mit Selfassessment (CME im SMF);

- elektronische Medien;

- Kombination Präsenz/Workshops:

- Printmedien,

- elektronische Medien.

Es sei darauf hingewiesen, dass ein Medienmix von Vorteil ist, da durch die geeigneten Medien Zeit- und Ortsunabhängigkeit des Lernens gewährleistet werden kann. $\mathrm{Zu}$ betonen ist auch, dass die Kommunikation bei der Fortbildung eine entscheidende Rolle spielt - Fortbildung darf nicht nur alleine in der Studierkammer stattfinden. Durch interaktive Fortbildungsformen kann einer möglichen Vereinsamung, ja einem Autismus, vorgebeugt werden, und die Fortzubildenden können ihre Erfahrungen und ihre Kenntnisse austauschen bzw. miteinander vergleichen.

Das Zielpublikum sind alle Facharzttitelträgerinnen und -träger. $\mathrm{Ob}$ es fachspezifischer Fortbildungsformen und -inhalte bedarf, diskutierten kürzlich in der SÄZ die Kollegen Gérard Waeber aus Lausanne und Peter Tschudi aus Basel [1]. Die Inhalte sollen auf das Zielpublikum ausgerichtet sein, die wissenschaftliche Basis muss 
immer dieselbe sein; die Evidenzgrade der Inhalte sollen explizit genannt werden.

Abschliessend seien noch einige Themata genannt, die aus meiner Sicht in der Fortbildung stärker gewichtet und somit auch in den Printmedien entsprechend dargestellt werden sollten:

- medizinisch-wissenschaftlich:

- Demographie,

- Epidemiologie,

- POL, klinische Vignetten, Kernbotschaften;

- Praxismanagement:

- E-Health,

- Gesundheitsökonomie,

- Ethik,

- Kommunikation;

- Behandlungsketten/Patientenpfade:

- Interprofessionalität;

- Skill-Mix oder Delegation/Substitution.

\section{Take Home Messages}

Als eine Art Quintessenz aus dem bisher Gesagten lassen sich folgende zentrale Anliegen ableiten:

- Dieselben praxisbezogenen, zukunftsorientierten Inhalte sollen modulartig in verschiedenen Medien präsentiert werden;

- es sollte eine Kooperation zwischen Printund elektronischen Medien, Moderatoren und grossen Weiterbildungszentren angestrebt werden;

- es sollten weitere Selbstevaluationsmittel erarbeitet und angeboten werden.

\section{Literatur}

1 Kesseli B. Fünf Fragen an Gérard Waeber und Peter Tschudi. Schweiz Ärztezeitung. 2008; 89(22):979-81. 